\title{
Radiation induced detwinning in nanotwinned $\mathrm{Cu}$
}

\author{
Y. Chen ${ }^{\mathrm{a}, \mathrm{b},{ }^{*}}$, H. Wang ${ }^{\mathrm{a}, \mathrm{c}}$, M.A. Kirk ${ }^{\mathrm{d}}$, M. $\mathrm{Li}^{\mathrm{d}}$, J. Wang ${ }^{\mathrm{e}}$ and X. Zhang ${ }^{\mathrm{a}, \mathrm{f}, \mathrm{g}, *}$ \\ ${ }^{a}$ Department of Materials Science and Engineering, Texas A\&M University, College Station, TX 77843- \\ 3123, USA \\ ${ }^{\mathrm{b}}$ MPA-CINT, Los Alamos National Laboratory, Los Alamos, NM 87545, USA \\ ${ }^{c}$ Department of Electrical and Computer Engineering, Texas A\&M University, College Station, TX \\ 77843-3128, USA \\ ${ }^{\mathrm{d}}$ Nuclear Engineering Division, Argonne National Laboratory, Argonne, Illinois 60439, USA \\ ${ }^{\mathrm{e}}$ Mechanical and Materials Engineering, University of Nebraska-Lincoln, Lincoln, NE 68588, USA \\ ${ }^{\mathrm{f}}$ Department of Mechanical Engineering, Texas A\&M University, College Station, TX 77843-3123, USA \\ ${ }^{g}$ School of Materials Engineering, Purdue University, West Lafayette, IN 47907, USA
}

\begin{abstract}
Superior radiation tolerance has been experimentally examined in nanotwinned metals. The stability of nanotwinned structure under radiation is the key factor for advancing the application of nanotwinned metals for nuclear reactors. We thus performed in situ radiation tests for nanotwinned $\mathrm{Cu}$ with various twin thicknesses inside a transmission electron microscope. We found that there is a critical twin thickness $(10 \mathrm{~nm})$, below which, radiation induced detwinning is primarily accomplished through migration of incoherent twin boundaries, in particular, detwinning is faster for thinner twins in this range, while above which, twins are stable.

*Corresponding authors. Emails: youxing@lanl.gov (Y.C.); xzhang98@purdue.edu (X.Z.)
\end{abstract}


Irradiation of metals results in abundant point defects [1-3] and their clusters [4-9], which cause severe void swelling and mechanical deterioration of materials [10-12]. Interfaces, such as grain boundaries (GBs) [13-17], heterophase interfaces [18-20] and free surfaces [21-23], have proven to be effective in alleviating radiation damage. Nanograined materials gather intense interest as a result of drastic enhancement of radiation tolerance shown both experimentally [15, 17] and theoretically [14, 24-27]. However, nanograins tend to coarsen rapidly at elevated temperature or under irradiation $[28,29]$, compromising the radiation tolerance of nanocrystalline materials. In contrast to nanograins, nanotwinned (nt) metallic materials with predominantly TBs have outstanding microstructral stability under both radiation [30] and annealing conditions [31, 32], as coherent twin boundaries (CTBs) are thermodynamically stable at elevated temperatures.

Recent studies show that CTBs can serve as defect sinks to reduce radiation damage in nt metals [33, 34]. In situ radiation studies and molecular dynamics (MD) simulations show that CTBs can effectively interact with radiation induced stacking fault tetrahedra (SFTs), resulting in the removal of SFTs in irradiated fcc metals [33-36]. Furthermore, deliberate introduction of nanovoids in conjunction with nanotwins establishes a network of defect sinks in nt metals. In situ TEM observation and atomistic simulations demonstrate that TBs can rapidly transport radiation induced defects to nanovoids, where defects are stored and annihilated, leading to significant enhancement of radiation tolerance in nt metals [37,38]. The stability of twins under radiation is thus one key factor in retaining the high radiation tolerance in nt metals. However, does radiation induce detwinning in nt metals?

Detwinning under mechanical deformation has been frequently observed in metals [33, 39-42] and the deformation induced detwinning mechanisms can be categorized to at least two 
cases. The first case is characterized by the migration of steps/dislocation disconnections at CTBs, where the steps derive from interaction between CTBs and inclined dislocations [43-48]. The second case is associated with the collective glide of Shockley partial dislocations at $\Sigma 3$ $\{112\}$ incoherent twin boundary (ITB) [49, 50]. It is anticipated that radiation induced detwinning could be accomplished through different mechanisms from deformation induced detwinning because the internal stress arisen from radiation process is not as significant as the case of mechanical deformation. In this paper, we studied the radiation response of epitaxial nt $\mathrm{Cu}$ with various twin thicknesses by using in situ $\mathrm{Kr}$ ion irradiation technique inside a transmission electron microscope. The migration of both CTBs and ITBs was investigated and the radiation induced detwinning mechanisms are discussed.

Epitaxial nt $\mathrm{Cu}$ films were deposited on $\mathrm{HF}$ etched $\mathrm{Si}$ (110) substrates using the magnetron sputtering technique at room temperature. The total film thickness was $\sim 1 \mu \mathrm{m}$ for $\mathrm{nt}$ $\mathrm{Cu}$. The deposition rate was $\sim 0.5 \mathrm{~nm} / \mathrm{s}$. Cross-sectional transmission electron microscopy (XTEM) samples were prepared by dimpling and low energy (3.5 keV) Ar ion milling and subsequent ion polishing. In situ $\mathrm{Kr}^{++}$ion irradiation at $1 \mathrm{MeV}$ was performed for $\mathrm{nt} \mathrm{Cu}$ at room temperature in the Intermediate Voltage Electron Microscope (IVEM) at Argonne National Laboratory, where an ion accelerator was attached to a HITACHI H-9000NAR transmission electron microscope. The microscope was operated at $200 \mathrm{kV}$ continuously during radiation in order to record the microstructural evolution. The average dose rate was $0.43-1.98 \times 10^{-3} \mathrm{dpa} \mathrm{s}^{-1}$. A CCD camera was used to capture microstructural evolution during radiation at 15 frame $\mathrm{s}^{-1}$. More details are referred to [34].

Detwinning occurs via migration of CTBs and ITBs during radiation. Fig. 1 compares snap shots of $\mathrm{nt} \mathrm{Cu}$ subjected to in situ $\mathrm{Kr}$ ion irradiation of up to 1.56 dpa to illustrate the ITB 
migration. The ITBs of thinner twins have higher tendency to migrate than those of thicker ones. As shown in Fig. 1, during radiation, the migration of ITBs occurs for the thinner twins (twin 1 and 3), whereas no significant ITB migration was observed for the thicker twin 2 . The average twin spacing slightly increased from $13 \mathrm{~nm}$ (at $0.01 \mathrm{dpa}$ ) to $15 \mathrm{~nm}$ after irradiation to $1.56 \mathrm{dpa}$, showing that fine twins have been eliminated.

One should note that in this study the definition of twins and matrices is relative to each other as the volume fraction of twins is very close to that of the matrix and the average twin thickness and matrix thickness is similar, $\sim 13 \mathrm{~nm}$. Irradiation of $\mathrm{nt} \mathrm{Cu}$ also induced a high density of stacking faults (SFs) decorating along the TBs. TEM micrographs in Fig. 2(a-b) compare the positions of CTBs at the same location of irradiated nt $\mathrm{Cu}$ before and after radiation to $1.56 \mathrm{dpa}$. The phase with bright contrast is defined as matrices and the dark regions as twins. Statistical studies (Fig. 2(c-d)) show the radiation induced the increase of the twin thickness and corresponding decrease in the matrix thickness, due to the migration of CTBs along its normal direction. Overall the thickness variation of twins and matrices (or the magnitude of CTB migration) shows little dependence on the original twin thickness. The thickness variations for both twins and matrices are close, $\sim 3 \mathrm{~nm}$ up to $1.56 \mathrm{dpa}$, and therefore the CTB migration speed is estimated to be $\sim 1 \mathrm{~nm} / \mathrm{dpa}$.

The prominent migration of thin twins leads to two interesting questions: How does ITB migrate? is there a quantitative relation between the migration of ITBs and twin thicknesses? Extensive studies have been performed to investigate migration of ITBs by analyzing the in situ radiation videos. A typical example shown in Fig. 3a captures the migration of four ITBs (T1-T4) with respective twin thicknesses of $2.8,1.4,5.2$ and $6.4 \mathrm{~nm}$. The migration traces of the four ITBs are shown in Fig. 3b. It is evident that ITBs migrate in a "stick-slip" fashion, i.e., the 
migration processes consist of instantaneous movement and dwelling. Fig. $3 \mathrm{~b}$ also shows that instantaneous migration speed could be as high as $21 \mathrm{~nm} / \mathrm{s}$. Fig. 4 shows ITB migration distances as a function of radiation dose with respect to twins with various thicknesses in the range of 2.8 $23 \mathrm{~nm}$. When twin thickness is below a critical value $(\sim 10 \mathrm{~nm})$, the velocity of ITB migration (described in unit of $\mathrm{nm} / \mathrm{dpa}$ ) decreases progressively with the increasing in twin thickness (Fig. 4b). Above the critical thickness, detwinning is not obviously observed.

Previous studies show that TBs in nt metals can actively engage and absorb radiation induced defect clusters, including dislocation loop and vacancy clusters [36, 37]. Our current study shows that both CTBs and ITBs not only interact with defects, but also migrate. In other words, the defect sinks (TBs) are mobile. CTBs migrate along <111> at a velocity of $\sim 1 \mathrm{~nm} / \mathrm{dpa}$ and its migration velocity is independent of twin thickness. This is different from detwinning associated with the migration of ITBs. In contrast, ITBs migrate along $\langle 112\rangle$ at a much faster velocity, varying from several to $60 \mathrm{~nm} / \mathrm{dpa}$. The migration of ITBs leads to detwinning. Two potential mechanisms may contribute to the migration of CTBs. First, radiation induced defects, such as dislocation loops and SFTs, accumulate and interact with CTBs, forming stacking fault ribbons at CTBs $[33,34]$. Second, steps/dislocation disconnections at CTBs that formed due to the interaction of CTBs and inclined dislocations could migrate along the direction normal to CTBs.

The high migration velocity of ITBs with small twin thickness ( $t$ ) is related to their defect structures. $\Sigma 3\{112\}$ ITB can be represented as an array of Shockley partial dislocations on each $\{111\}$ plane, containing three repetitive partial dislocations: $\mathbf{b}_{1}=1 / 6[11 \overline{2}], \mathbf{b}_{2}=1 / 6[\overline{2} 11]$ and $\left.\mathbf{b}_{3}=1 / 6[1 \overline{2} 1]\right)$, where $\mathbf{b}_{1}$ is an edge partial dislocation and $\mathbf{b}_{\mathbf{2}}$ and $\mathbf{b}_{\mathbf{3}}$ are mixed partial dislocations with screw components of opposite sign. Without radiation, the ITBs with the thicknesses of 2.8 
- $23 \mathrm{~nm}$ are stable at room temperature, as demonstrated in our previous study [49] in $\mathrm{Cu}$ that ITBs are stable when the twin thickness is larger than $1.9 \mathrm{~nm}$. Energetically ITBs tend to migrate to reduce the area of CTBs (and consequent energy stored in CTBs) with the driving force $F$ $\left(=2 \gamma_{T}\right.$, where $\gamma_{T}$ is excess energy of CTB). We note that friction force $\left(F_{P}\right.$, or Peierls barrier $)$, for the migration of ITBs increases with the twin thickness, as partial dislocations in the ITB migrate collectively [49]. When the driving force is larger than the friction stress $\left(F>F_{P}\right)$, ITB could migrate to reduce the area of CTBs (case 1 in Fig. 4b); when $F<F_{P}$, ITB would not migrate. Fig. $4 \mathrm{~b}$ shows that when $\mathrm{t}>10 \mathrm{~nm}$, ITBs barely migrate, suggesting that the critical thickness $\left(\mathrm{t}^{*}\right)$ for ITB migration is $\sim 10 \mathrm{~nm}$. When $\mathrm{t}>\mathrm{t}^{*}$, detwinning could begin from the corner (case 2 in Fig. 4b). The driving force for ITB migration in case 2 is negligible as the detwinning does not change the total area of CTBs (does not reduce the energy stored at TBs). A further increase of twin thickness would make segment of ITBs to migrate randomly (case 3 in Fig. 4b), that is in this case both twinning and detwinning may occur, but both process would increase the area of CTBs. The direction of ITB migration is random as such is mainly determined by both radiation induced local shear stress [49] and the density and type of radiation induced defects across the ITBs [51].

In contrast, when $t<t^{*}$, the migration velocity of TBs decreases with the increase of twin thickness, which is rationalized as follows. During radiation, the driving force on individual Shockley partial dislocation in the ITB can be treated as $F_{1}=F / L=2 \gamma_{T} / L$. ( $L$ is the number of atomic layers of the twin, $L=t / d_{111}, d_{111}$ is the interplanar spacing of $\{111\}$ planes $)$. For fcc and hcp metals, the dislocation velocity and applied stress follows $v=A \tau^{m}[52]$, where A is a material constant, $m$ is approximately 1 at $300 \mathrm{k}$ in pure metals. Therefore, the velocity of ITB migration can be rationalized as $v=A \tau=\frac{A F_{1}}{b}=B / t(b$ is the magnitude of Burgers vector of 
partial dislocation) which qualitatively explains the trend that the migration velocity decreases with the increase of twin thickness when $t<t^{*}$.

It is noted that there is scattering for data points, particularly for ITBs with small thicknesses. One possibility is the variation of the TEM foil thickness across the wedge sample. The driving force discussed previously is evaluated thermodynamically. During in situ radiation, a kinetic process is also involved, that is the migration of different segments on a dislocation line may not occur simultaneously. ITBs may migrate at a slower speed when the TEM foil becomes thicker, causing the scattering of data in Fig. $4 \mathrm{~b}$.

It is worth pointing out that ITB migration velocity also depends on stacking fault energy (SFE) [49]. Our current study only discussed detwinning in $\mathrm{Cu}$ with low SFE. For ITBs in metal with high SFE, the driving force $\left(2 \gamma_{T} / b L\right)$ for ITB migration is large. However, the Peierls barrier associated with the three TDs in metal with high SFE is also high as high SFE prevents the dissociation of the three TDs at ITBs [53]. Therefore, future experiments are required to examine the ITB response under radiation in metal with high SFE.

In summary, detwinning occurs in irradiated nanotwinned $\mathrm{Cu}$ via migration of both ITB and CTB during radiation. In particular, the migration speed of CTBs is sluggish, $\sim 1 \mathrm{~nm} / \mathrm{dpa}$, independent of twin thickness. In comparison, ITBs migrate aggressively at a much greater speed when the twin thickness is below $10 \mathrm{~nm}$. The migration speed of ITBs decreases progressively with increasing twin thickness, while ITBs with large twin thickness are stable during radiation. The ITB migration consists of instantaneous movement and dwelling during radiation. Having mobile ITBs in irradiated metallic materials could be beneficial to enhance radiation tolerance, as these mobile defect sinks can have a larger territory to capture, trap and eliminate radiation 
induced defects. However it is also important to retain a high density of ITBs so that these defects sinks can operate over a long period of time to eliminate radiation induced defects.

We acknowledge financial support by NSF-DMR-Metallic Materials and Nanostructures Program under grant no. 1304101. The electron microscopy with in situ ion irradiation was accomplished at Argonne National Laboratory at the IVEM-Tandem Facility, a U.S. Department of Energy Facility funded by the DOE Office of Nuclear Energy, operated under Contract No. DE-AC02-06CH11357 by U Chicago, Argonne, LLC. We also acknowledge the use of microscopes at the Microscopy and Imaging Center at Texas A\&M University and the DOE Center for Integrated Nanotechnologies managed by Los Alamos National Laboratory.

1. G. D. Watkins, EPR Observation of Close Frenkel Pairs in Irradiated ZnSe, Phys. Rev. Lett. 33 (1974) 223.

2. B. D. Wirth, How Does Radiation Damage Materials?, Science 318 (2007) 923.

3. C.-C. Fu, J. D. Torre, F. Willaime et al., Multiscale modelling of defect kinetics in irradiated iron, Nat. Mater. 4 (2005) 68.

4. S. J. Zinkle, L. E. Seitzman,W. G. Wolfer, I. Energy calculations for pure metals, Philos. Mag. A 55 (1987) 111.

5. B. N. Singh,S. J. Zinkle, Defect accumulation in pure fcc metals in the transient regime: a review, J. Nucl. Mater. 206 (1993) 212.

6. K. Arakawa, K. Ono, M. Isshiki et al., Observation of the one-dimensional diffusion of nanometer-sized dislocation loops, Science 318 (2007) 956.

7. Y. Matsukawa,S. J. Zinkle, One-dimensional fast migration of vacancy clusters in metals, Science 318 (2007) 959.

8. C. Cawthorne,E. J. Fulton, Voids in Irradiated Stainless Steel, Nature 216 (1967) 575.

9. Y. N. Osetsky, D. J. Bacon, B. N. Singh et al., Atomistic study of the generation, interaction, accumulation and annihilation of cascade-induced defect clusters, J. Nucl. Mater. 307-311, Part 2 (2002) 852.

10. L. K. Mansur, Theory and experimental background on dimensional changes in irradiated alloys, J. Nucl. Mater. 216 (1994) 97.

11. F. A. Garner, M. B. Toloczko,B. H. Sencer, Comparison of swelling and irradiation creep behavior of fcc-austenitic and bcc-ferritic/martensitic alloys at high neutron exposure, J. Nucl. Mater. 276 (2000) 123.

12. D. Kiener, P. Hosemann, S. A. Maloy et al., In situ nanocompression testing of irradiated copper, Nat. Mater. 10 (2011) 608. 
13. B. N. Singh, Effect of grain size on void formation during high-energy electron irradiation of austenitic stainless steel, Philos. Mag. 29 (1974) 25.

14. B. N. Singh, M. Eldrup, S. J. Zinkle et al., On grain-size-dependent void swelling in pure copper irradiated with fission neutrons, Philos. Mag. A 82 (2002) 1137.

15. S. Ukai,M. Fujiwara, Perspective of ODS alloys application in nuclear environments, J. Nucl. Mater. 307-311, Part 1 (2002) 749.

16. W. Z. Han, M. J. Demkowicz, E. G. Fu et al., Effect of grain boundary character on sink efficiency, Acta Mater. 60 (2012) 6341.

17. C. Sun, M. Song, K. Y. Yu et al., In situ evidence of defect cluster absorption by grain boundaries in Kr Ion irradiated nanocrystalline Ni, Metall. Mater. Trans. A 44 (2013) 1966.

18. M. J. Demkowicz, R. G. Hoagland,J. P. Hirth, Interface structure and radiation damage resistance in Cu-Nb multilayer nanocomposites, Phys. Rev. Lett. 100 (2008) 136102.

19. E. G. Fu, A. Misra, H. Wang et al., Interface enabled defects reduction in helium ion irradiated $\mathrm{Cu} / \mathrm{V}$ nanolayers, J. Nucl. Mater. 407 (2010) 178.

20. A. Misra, M. J. Demkowicz, X. Zhang et al., The radiation damage tolerance of ultra-high strength nanolayered composites, JOM 59 (2007) 62.

21. E. M. Bringa, J. D. Monk, A. Caro et al., Are Nanoporous Materials Radiation Resistant?, Nano Lett. 12 (2011) 3351.

22. E. Fu, M. Caro, L. Zepeda-Ruiz et al., Surface effects on the radiation response of nanoporous Au foams, Appl. Phys. Lett. 101 (2012) 191607.

23. C. Sun, D. Bufford, Y. Chen et al., In situ study of defect migration kinetics in nanoporous Ag with enhanced radiation tolerance, Sci. Rep. 4 (2014) 3737.

24. M. Samaras, P. M. Derlet, H. Van Swygenhoven et al., Computer simulation of displacement cascades in nanocrystalline Ni, Phys. Rev. Lett. 88 (2002) 125505.

25. X.-M. Bai, A. F. Voter, R. G. Hoagland et al., Efficient annealing of radiation damage near grain boundaries via interstitial emission, Science 327 (2010) 1631.

26. D. Chen, J. Wang, T. Chen et al., Defect annihilation at grain boundaries in alpha-Fe, Sci. Rep. 3 (2013) 1450.

27. P. C. Millett, A. El-Azab, S. Rokkam et al., Phase-field simulation of irradiated metals: Part I: Void kinetics, Comput. Mater. Sci. 50 (2011) 949.

28. G. Ackland, Controlling Radiation Damage, Science 327 (2010) 1587.

29. D. Kaoumi, A. T. Motta,R. C. Birtcher, A thermal spike model of grain growth under irradiation, J. Appl. Phys. 104 (2008) 073525.

30. M. J. Demkowicz, O. Anderoglu, X. Zhang et al., The influence of $\Sigma 3$ twin boundaries on the formation of radiation-induced defect clusters in nanotwinned Cu, J. Mater. Res. 26 (2011) 1666.

31. X. Zhang,A. Misra, Superior thermal stability of coherent twin boundaries in nanotwinned metals, Scripta Mater. 66 (2012) 860.

32. O. Anderoglu, A. Misra, H. Wang et al., Thermal stability of sputtered Cu films with nanoscale growth twins, J. Appl. Phys. 103 (2008) 094322.

33. K. Yu, D. Bufford, C. Sun et al., Removal of stacking-fault tetrahedra by twin boundaries in nanotwinned metals, Nat. Commun. 4 (2013) 1377.

34. Y. Chen, J. Li, K. Yu et al., In situ studies on radiation tolerance of nanotwinned Cu, Acta Mater. 111 (2016) 148.

35. M. Niewczas,R. G. Hoagland, Molecular dynamic studies of the interaction of $a / 6<112>$ Shockley dislocations with stacking fault tetrahedra in copper. Part II: Intersection of stacking fault tetrahedra by moving twin boundaries, Philos. Mag. 89 (2009) 727.

36. J. Li, K. Y. Yu, Y. Chen et al., In situ study of defect migration dinetics and self-healing of twin boundaries in heavy ion irradiated nanotwinned metals, Nano Lett. 15 (2015) 2922. 
37. Y. Chen, K. Y. Yu, Y. Liu et al., Damage-tolerant nanotwinned metals with nanovoids under radiation environments, Nat. Commun. 6 (2015) 7036.

38. Y. Chen, X. Zhang,J. Wang, Radiation Enhanced Absorption of Frank Loops by Nanovoids in $\mathrm{Cu}$, JOM 68 (2016) 235.

39. O. Anderoglu, A. Misra, J. Wang et al., Plastic flow stability of nanotwinned Cu foils, Int. J. Plast. 26 (2010) 875.

40. Y. Wei, The kinetics and energetics of dislocation mediated de-twinning in nano-twinned facecentered cubic metals, Mater. Sci. Eng., A 528 (2011) 1558.

41. A. Hodge, T. Furnish, C. Shute et al., Twin stability in highly nanotwinned Cu under compression, torsion and tension, Scripta Mater. 66 (2012) 872.

42. Y. Liu, J. Jian, Y. Chen et al., Plasticity and ultra-low stress induced twin boundary migration in nanotwinned Cu by in situ nanoindentation studies, Appl. Phys. Lett. 104 (2014) 231910.

43. C. Hong, N. Tao, X. Huang et al., Nucleation and thickening of shear bands in nano-scale twin/matrix lamellae of a Cu-Al alloy processed by dynamic plastic deformation, Acta Mater. 58 (2010) 3103.

44. N. Li, J. Wang, A. Misra et al., Twinning dislocation multiplication at a coherent twin boundary, Acta Mater. 59 (2011) 5989.

45. S. Ni, Y. Wang, X. Liao et al., Effect of grain size on the competition between twinning and detwinning in nanocrystalline metals, Phys. Rev. B 84 (2011) 235401.

46. S. Ni, Y. Wang, X. Liao et al., The effect of dislocation density on the interactions between dislocations and twin boundaries in nanocrystalline materials, Acta Mater. 60 (2012) 3181.

47. Y. Cao, Y. Wang, Z. Chen et al., De-twinning via secondary twinning in face-centered cubic alloys, Mater. Sci. Eng., A 578 (2013) 110.

48. Y. M. Wang, F. Sansoz, T. LaGrange et al., Defective twin boundaries in nanotwinned metals, Nat. Mater. 12 (2013) 697.

49. J. Wang, N. Li, O. Anderoglu et al., Detwinning mechanisms for growth twins in face-centered cubic metals, Acta Mater. 58 (2010) 2262.

50. J. Wang, A. Misra,J. Hirth, Shear response of $\Sigma 3\{112\}$ twin boundaries in face-centered-cubic metals, Phys. Rev. B 83 (2011) 064106.

51. N. Li, J. Wang, Y. Q. Wang et al., Incoherent twin boundary migration induced by ion irradiation in $\mathrm{Cu}$, J. Appl. Phys. 113 (2013) 023508.

52. D. Hull,D. J. Bacon, Introduction to dislocations, Butterworth-Heinemann, 2001.

53. J. Wang, O. Anderoglu, J. Hirth et al., Dislocation structures of S3 $\{112\}$ twin boundaries in face centered cubic metals, Appl. Phys. Lett. 95 (2009) 021908. 


\section{Figure captions}

Fig.1. (a-d) TEM studies on migration of incoherent twin boundaries (ITBs) in nanotwinned Cu subjected to in situ $\mathrm{Kr}$ ion irradiation of up to $1.56 \mathrm{dpa}$. Three typical ITBs are labeled with respective twin thickness of 6, 23 and $8 \mathrm{~nm}$ in (a). During radiation, ITB migration occurs for the thinner twins (twin 1 and 3), whereas no significant ITB migration was observed for the thicker twin 2. (e-f) Comparison of the distribution of twin spacings shows that the average twin spacing slightly increased from $13 \mathrm{~nm}$ (at 0.01 dpa) to $15 \mathrm{~nm}$ after irradiation to $1.56 \mathrm{dpa}$.

Fig. 2. In situ study on radiation induced CTB migration. (a-b) TEM snap shots document the positions of CTBs at the same location before and after radiation to $1.56 \mathrm{dpa}$. The bright and dark areas correspond to the matrix and twin, respectively. (c-d) Statistical studies show that radiation induced the increase of the twin thickness and decrease in the matrix thickness, due to migration of CTB along its normal direction. Overall the thickness variation of twins and matrices (or the magnitude of CTB migration) shows little dependence on the original twin thickness. The thickness variations for both twins and matrices are close, $\sim 3 \mathrm{~nm}$ up to $1.56 \mathrm{dpa}$, leading to an average CTB migration speed of $\sim 1 \mathrm{~nm} / \mathrm{dpa}$.

Fig. 3. In situ TEM snap shots showing the migration of ITBs consisting of instantaneous ITB movement and dwelling during radiation. Four ITBs (T1-T4) with respective thicknesses of 2.8, 1.4, 5.2 and $6.4 \mathrm{~nm}$ were selected to track their migration processes. At $0.07 \mathrm{~s}$, the tip of T1 became blunted. At $0.14 \mathrm{~s}$, T1 migrated rightward by $1 \mathrm{~nm}$. At $0.21 \mathrm{~s}$ and $0.28 \mathrm{~s}$, T1 migrated by another 0.5 and $1.5 \mathrm{~nm}(1.5 \mathrm{~nm}$ and 3 $\mathrm{nm}$ away from initial position). T1 then stalled afterwards. Meanwhile T2 migrated over $1 \mathrm{~nm}$ from 0.21 to $0.28 \mathrm{~s}$, while T3 and T4 did not migrate. (b) Plots of ITB migration distance vs. accumulative time for the four ITBs show that stick-slip type of ITB migrations consisting of instantaneous movement and dwelling of the ITBs. Instantaneous migration speed could be as high as $21 \mathrm{~nm} / \mathrm{s}$.

Fig. 4. The dependence of ITB migration process on twin thickness. (a) The plot of ITB migration distance over radiation dose for numerous twins with twin thickness of $2.8-23 \mathrm{~nm}$ shows that in general the thinner ITBs have greater migration distance. (b) The velocity of ITBs in unit of $\mathrm{nm} / \mathrm{dpa}$ decreases progressively with the increasing twin thickness. The critical film thickness, $\mathrm{t}^{*}$, is $\sim 10 \mathrm{~nm}$. For case 1 $\left(\mathrm{t}<\mathrm{t}^{*}\right)$, detwinning reduces the area of CTBs and thinner twins appear to migrate at a greater velocity. For case 2, when $t$ is moderately greater than $10 \mathrm{~nm}$, detwinning doesn't change the area of CTBs, and hence TB migration is less likely; In case 3, both twinning and detwinning would increase the area of CTBs, reducing the likely for ITB migration. 

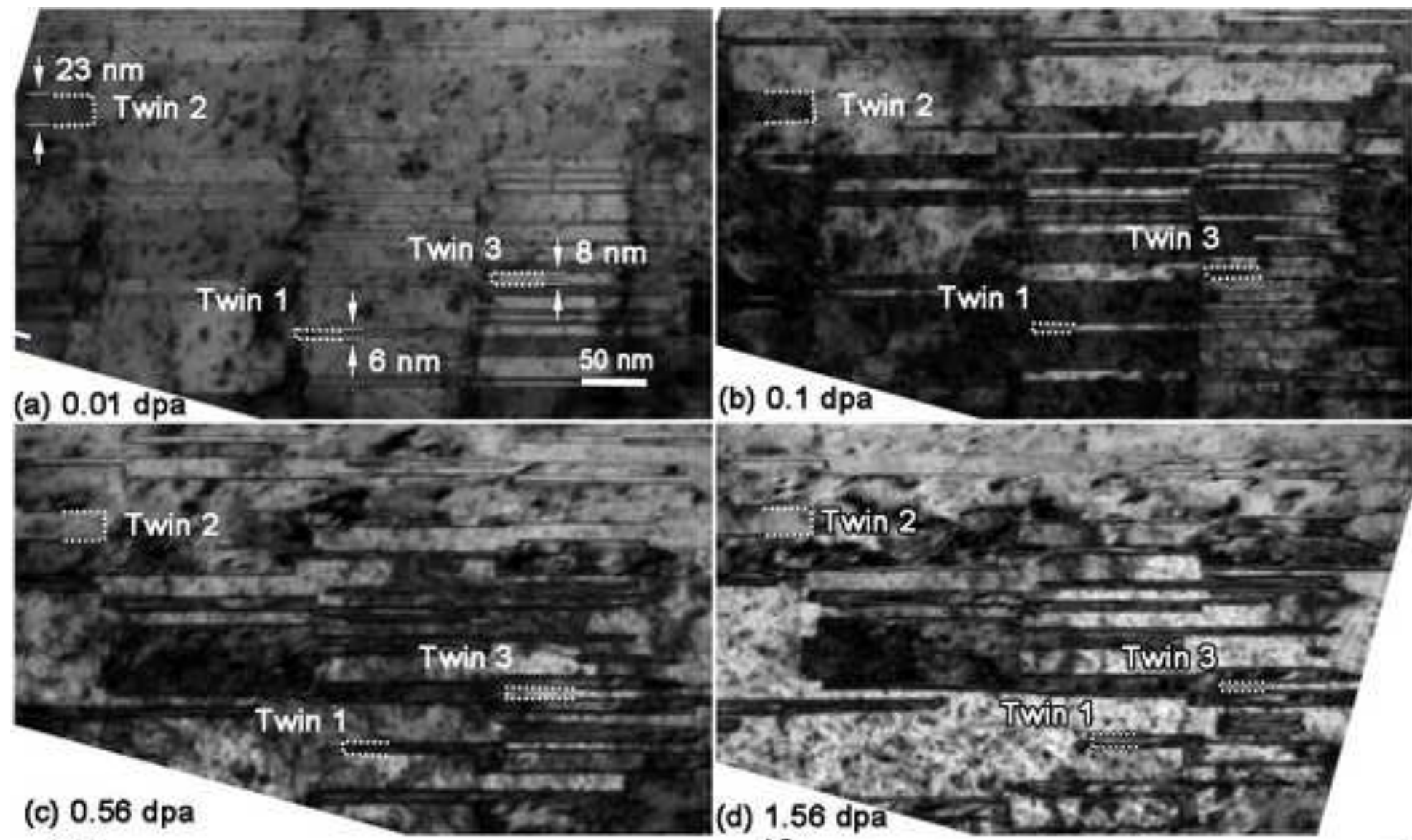

(b) $0.1 \mathrm{dpa}$
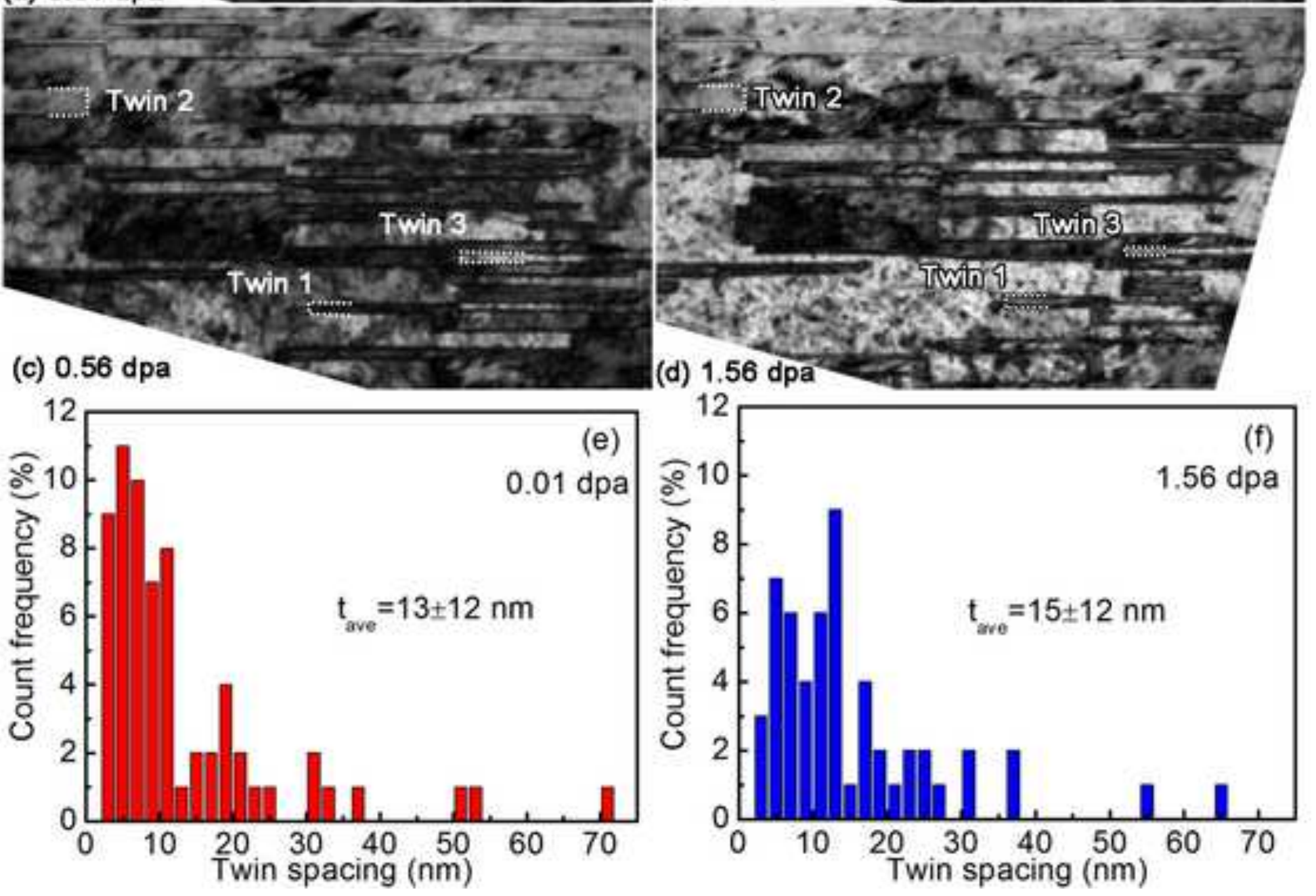
(a) $0 \mathrm{dpa}$

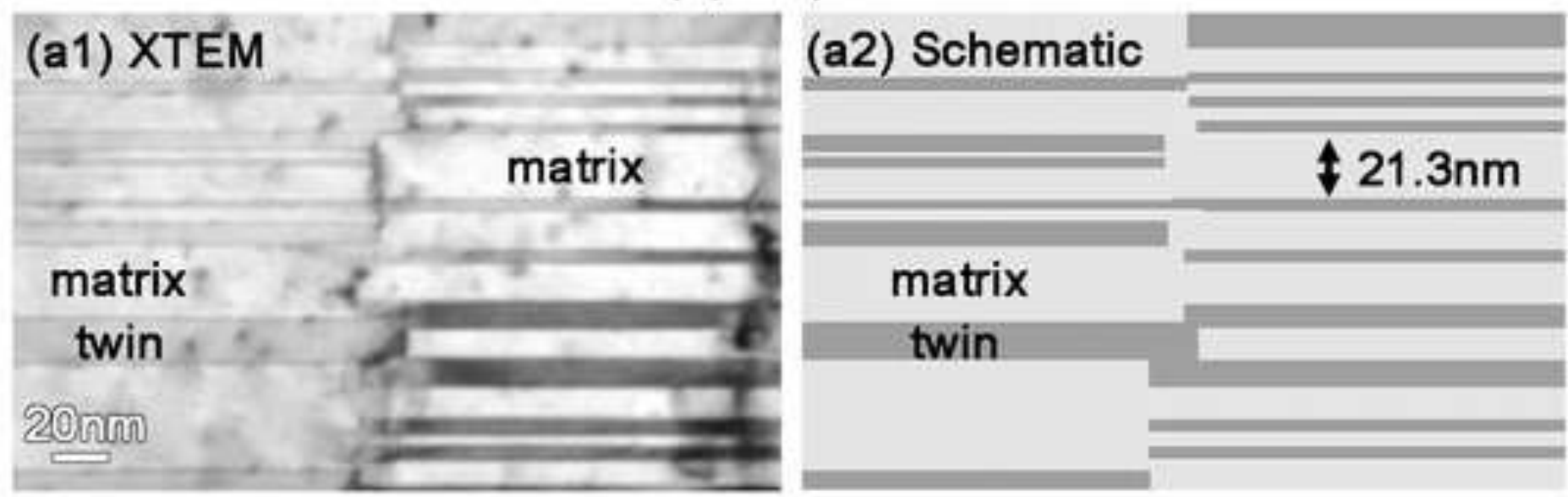

(b) $1.56 \mathrm{dpa}$

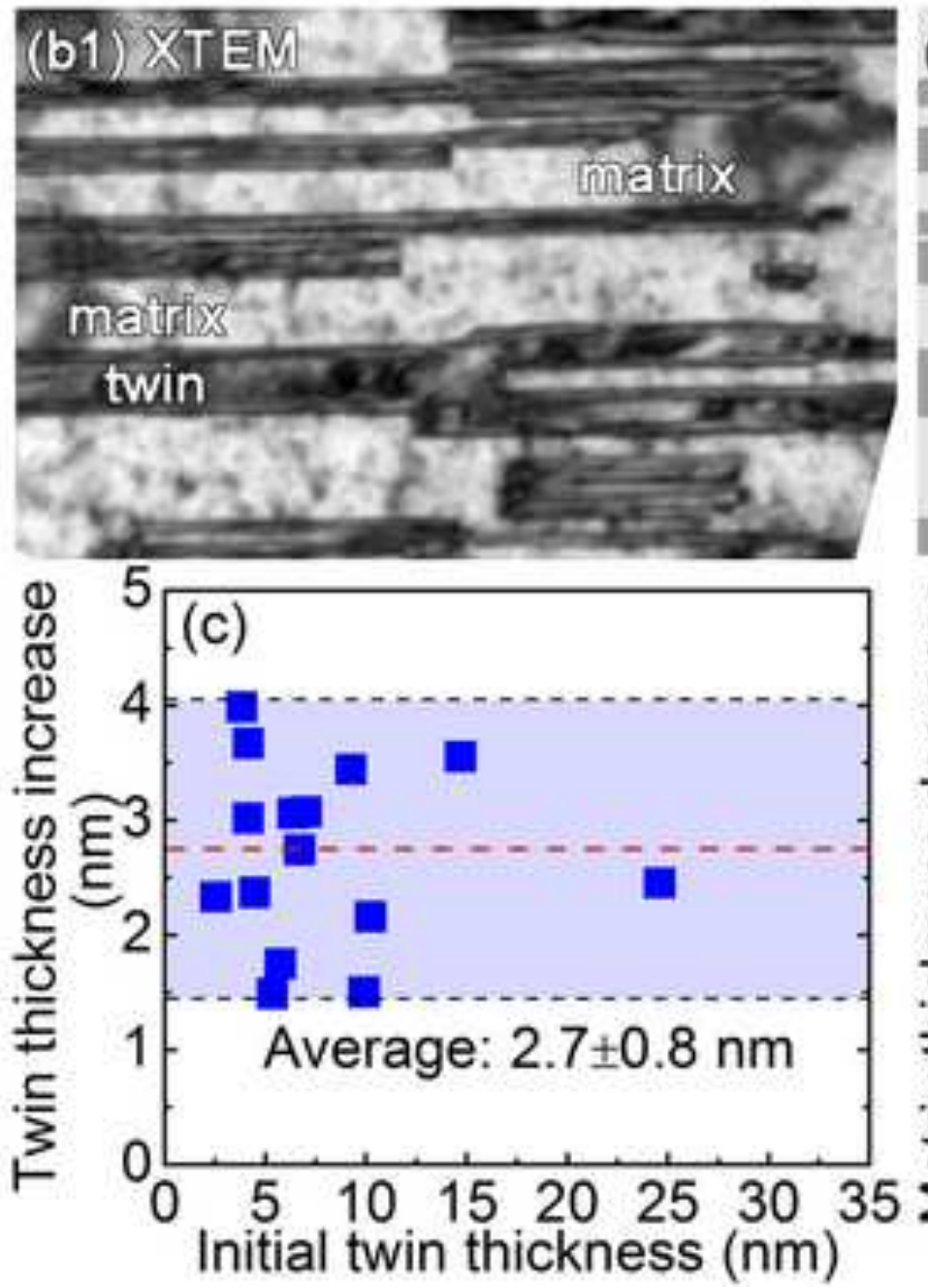

(b2) Schematic

matrix twin

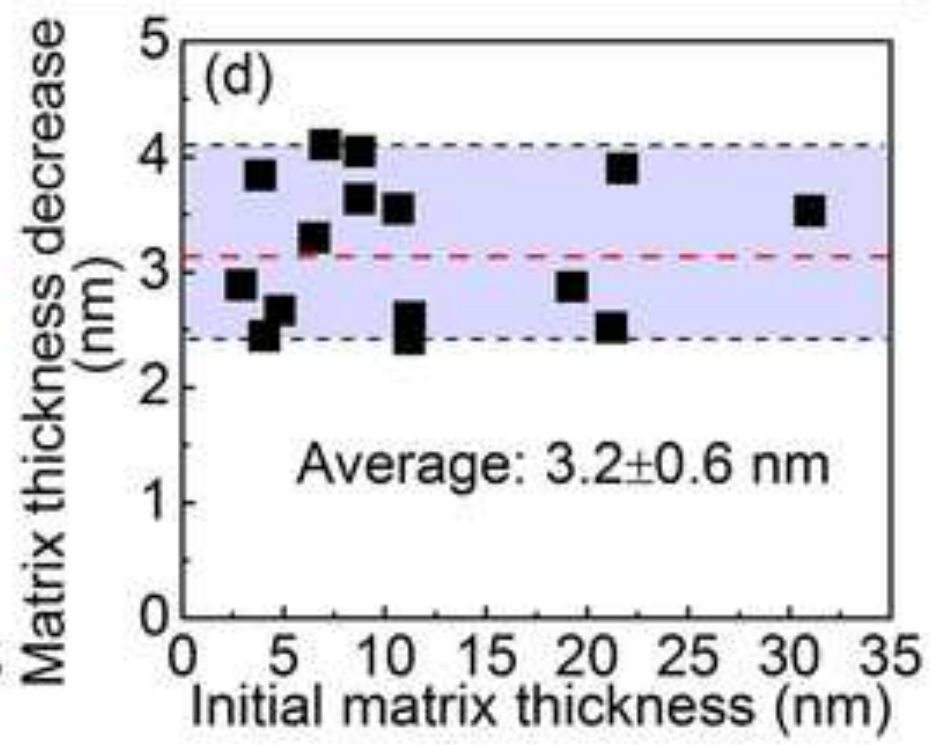




\section{(a) Instantaneous ITB migration}
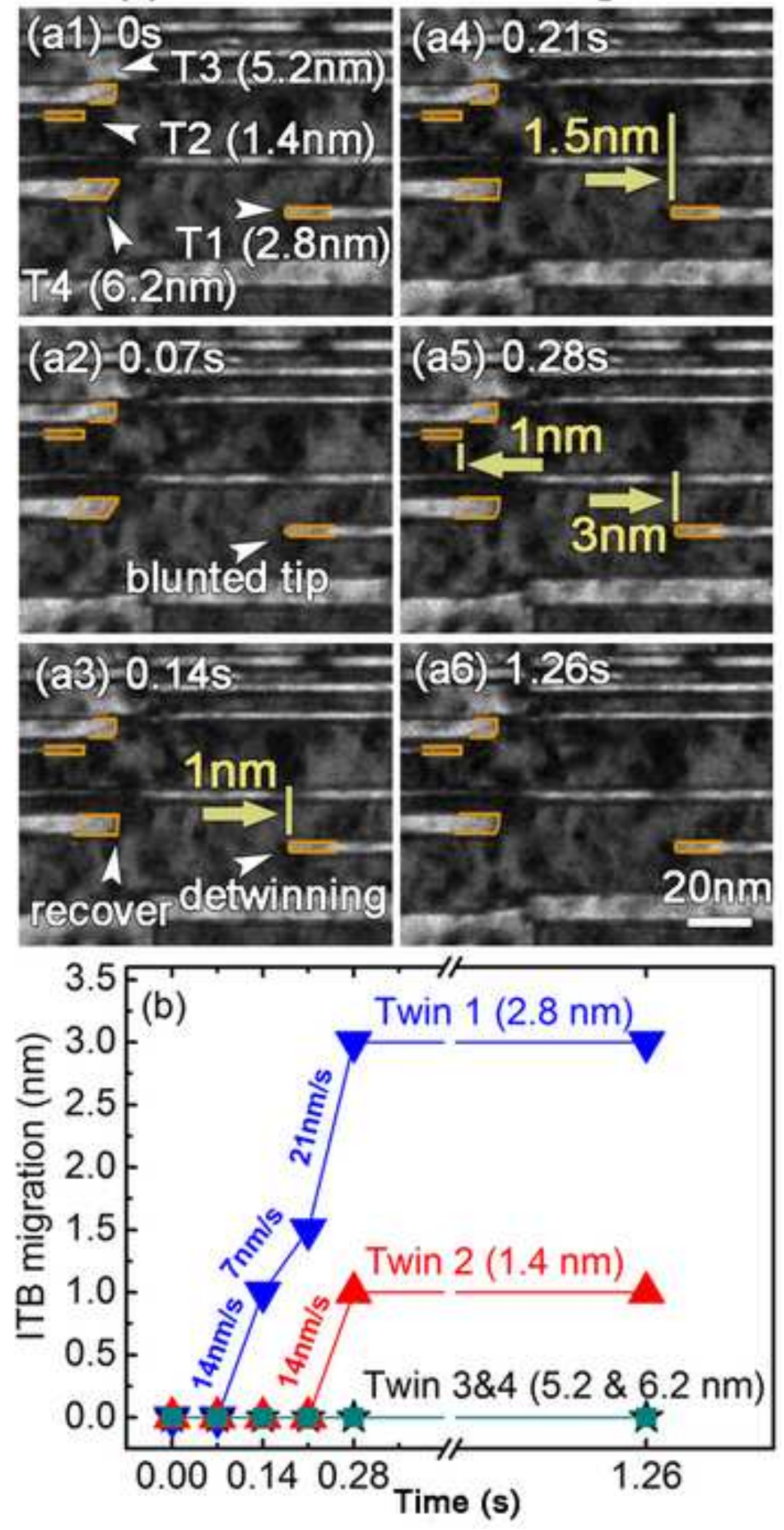
Time (s)
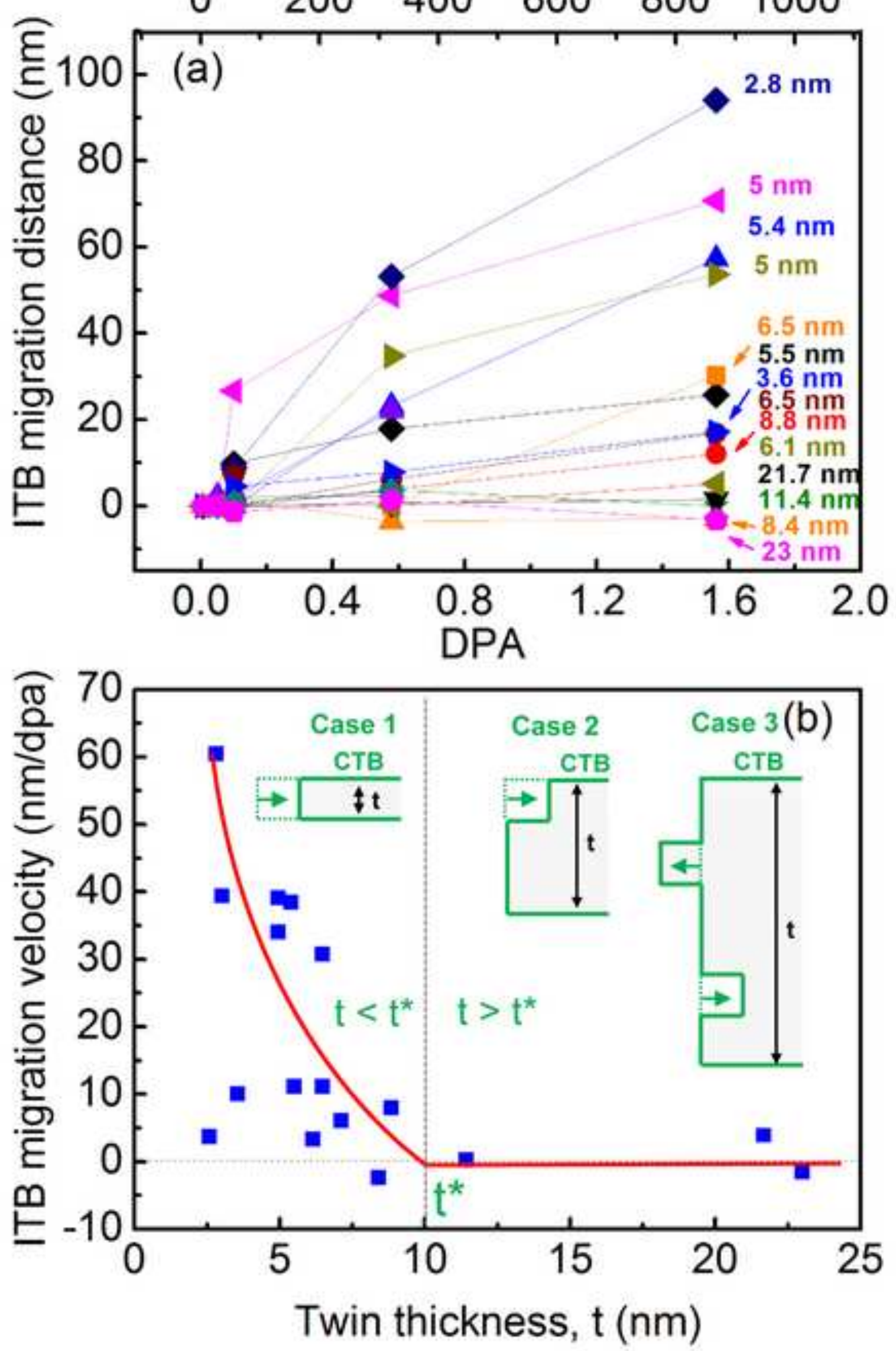
(a) $0 \mathrm{dpa}$

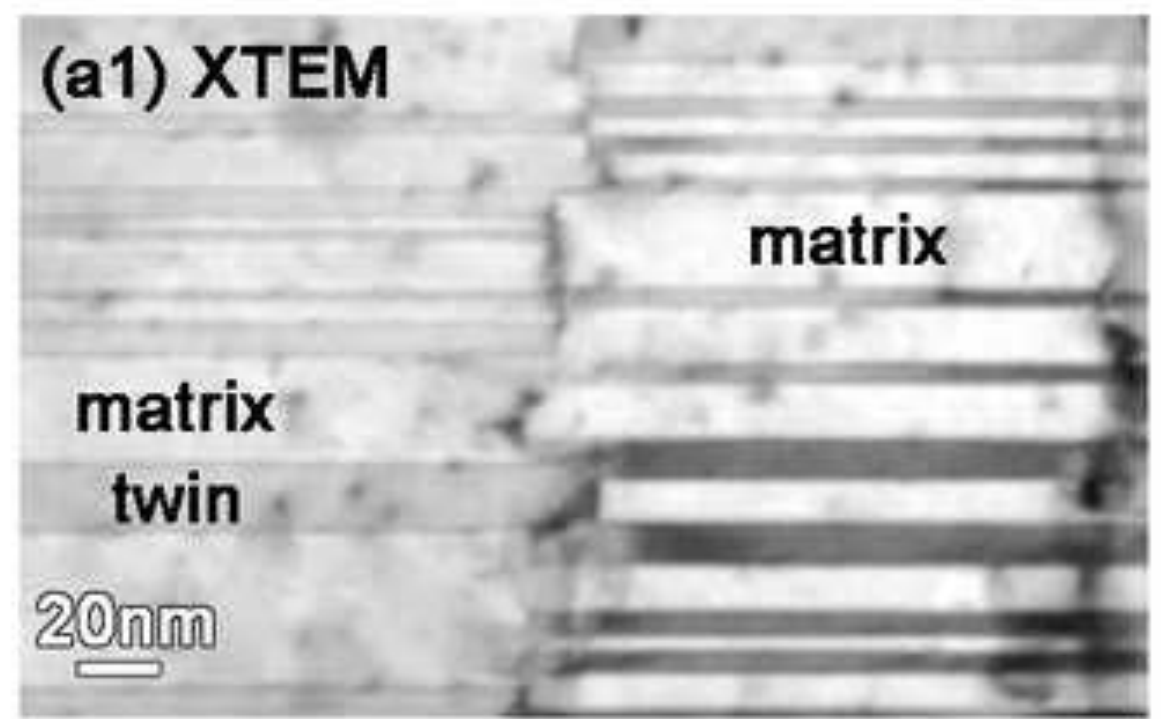

\section{(a2) Schematic}

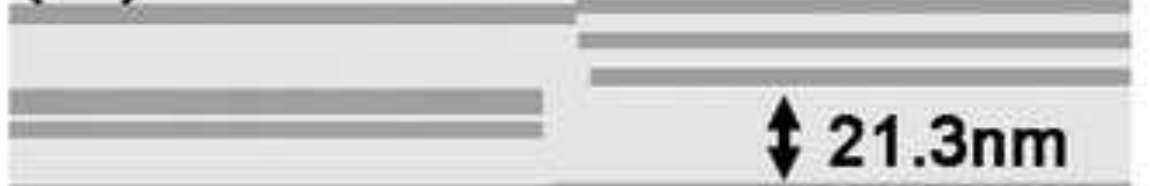

(b) $1.56 \mathrm{dpa}$
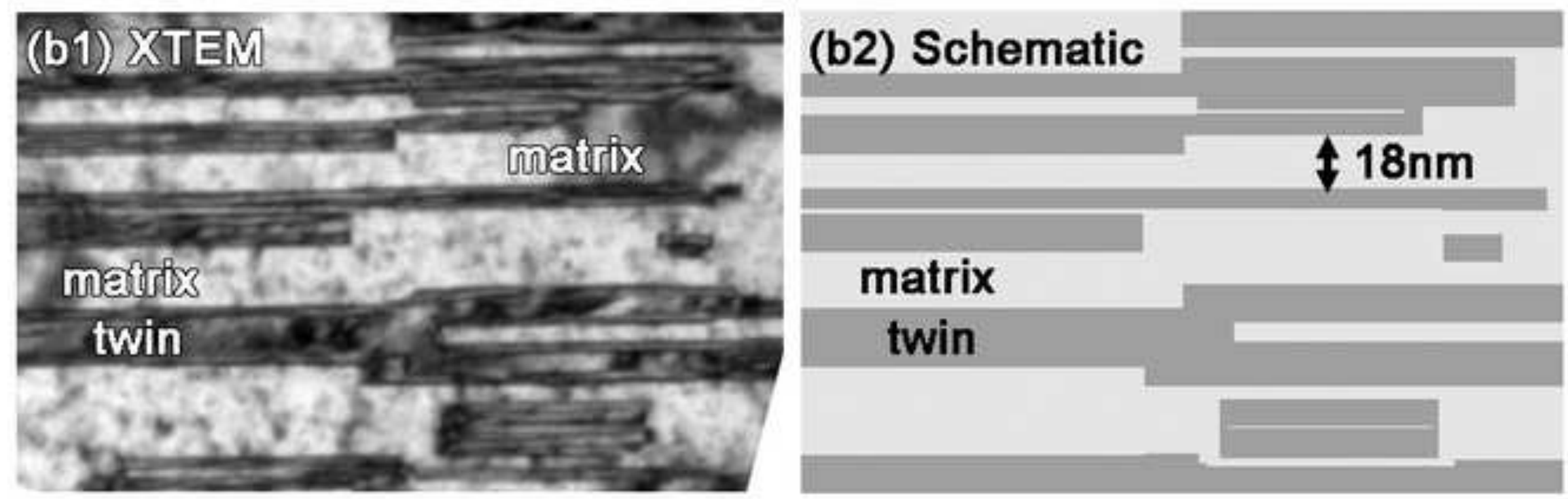